BULLETIN Bulletin hispanique

HISPANIQUE Université Michel de Montaigne Bordeaux

121-1 | 2019

La épica en el mundo hispánico (Siglo de Oro)

Variations sur le Siècle d'Or : Bataillon, Cassou, Rumeau, Aubrun, Molho. Mercedes BLANCO et Maria ZERARI (dir.)

Paris, Éditions Hispaniques, Coll. « Littérature Hispanophone médiévale et classique ", 2018

Nadine Ly Aguila

\title{
OpenEdition
}

Journals

Édition électronique

URL : https://journals.openedition.org/bulletinhispanique/8297

DOI : 10.4000/bulletinhispanique.8297

ISSN : 1775-3821

Éditeur

Presses universitaires de Bordeaux

Édition imprimée

Date de publication : 24 juin 2019

Pagination : 400-408

ISBN : 979-10-300-0363-5

ISSN : 0007-4640

Référence électronique

Nadine Ly Aguila, "Variations sur le Siècle d'Or: Bataillon, Cassou, Rumeau, Aubrun, Molho. Mercedes blanco et Maria zerari (dir.) », Bulletin hispanique [En ligne], 121-1 | 2019, mis en ligne le 24 juin 2019, consulté le 07 janvier 2023. URL : http://journals.openedition.org/bulletinhispanique/8297 ; DOI : https://doi.org/10.4000/bulletinhispanique.8297

Ce document a été généré automatiquement le 7 janvier 2023.

Tous droits réservés 


\section{Variations sur le Siècle d'Or: Bataillon, Cassou, Rumeau, Aubrun, Molho. Mercedes BLANCO et Maria ZERARI (dir.)}

Paris, Éditions Hispaniques, Coll. « Littérature Hispanophone médiévale et classique », 2018

\section{Nadine Ly Aguila}

\section{RÉFÉRENCE}

Variations sur le Siècle d'Or : Bataillon, Cassou, Rumeau, Aubrun, Molho. Mercedes BLANCo et Maria ZERARI (dir.), Paris, Éditions Hispaniques, Coll. « Littérature Hispanophone médiévale et classique », 2018, 215 p., ISBN : 978-2-85355-098-7

1 Si le titre de l'ouvrage fait littéralement écho - à l'exception de l'article, qui change tout - à un sous-titre («Variations sur un Siècle d'Or ») de l'essai que Jacques Lafaye composait, après le décès du Maître, en hommage à Marcel Bataillon ${ }^{1}$, l'illustration de couverture, "Le concert des anges » du Gréco et l'épigraphe de Stockhausen qui ouvre la préface: «La variation c'est plusieurs objets différents présentés sous la même lumière qui les traverse ", placent d'emblée le volume sous le double éclairage de la peinture et de la musique. Le peintre réapparaîtra dans la deuxième Variation dédiée à Jean Cassou, dans la belle analyse que Maria Zerari propose de son essai sur le Gréco (p. 97-112). Quant à la musique, on la retrouve, en forme de scherzo verbal, à l'ouverture de la cinquième, "Les variations Molho » (p. 159) dont le titre fait de l'hispaniste la figure même de la ou des variations.

2 Pour le livre de 2018, c'est le Siècle d'Or, pris dans la variété de son exceptionnel chatoiement, qu'illuminent différemment les travaux des cinq hispanistes ici honorés par les variations de six auteurs, spécialistes du Siècle d'Or: Mercedes Blanco pour M. 
Bataillon (45 p.), Maria Zerari pour J. Cassou (49 p.), Roland Béhar pour A. Rumeau (25 p.), Florence d'Artois et Yves Germain pour C.V. Aubrun (12 p.), Jean-Pierre Étienvre pour M. Molho (11 p.). Les textes sont suivis d'une série d'Illustrations : photos des cinq hispanistes, lettres, couvertures des premières éditions d'ouvrages ... La Préface (par M. Blanco et M. Zerari) rend hommage aux pré-fondateurs de l'Institur d'Études Hispaniques (p.12-16) et elle inscrit la fondation de l'I.E.H. dans une histoire plus générale de l'hispanisme français en signalant l'existence, à Toulouse et à Bordeaux, des deux premières chaires d'Espagnol. Une longue et riche collection de notes, présentes tout au long de l'ouvrage, rappelle l'histoire de cette histoire. Enfin, on y voit que trois de ces grands hispanistes ont été professeurs à la Faculté des Lettres de Bordeaux (Bataillon au Lycée de Talence ...) avant d'entrer en Sorbonne et que trois d'entre eux ont dirigé le Bulletin Hispanique: le rôle de la revue a été décisif dans la diffusion des travaux des premiers hispanistes et le développement de l'hispanisme français ${ }^{2}$.

3 Dans une étude aussi ample que profonde, aussi précise et documentée qu'inspirée, «Marcel Bataillon: méditations sur la littérature» (p.27-71), organisée en trois parties (I- Érudition et engagement entre deux guerres; II - L'érasmisme, un objet historique ou un objet littéraire; III - Cours de littérature classique par temps de guerre), Mercedes Blanco définit doublement Bataillon comme "parfait érudit » mais aussi comme un «intellectuel, quelqu'un pour qui l'activité de recherche et d'enseignement impliquait des responsabilités éthiques et politiques» (p. 34). Ce sont ces deux qualités, incarnées au plus haut point par le "Prince des hispanistes » et, j'ajoute, par elle-même, qui vont guider toutes ses analyses, depuis celle de la grande thèse : Érasme et l'Espagne. Recherches sur l'histoire spirituelle du XVI siècle (1937) jusqu'aux travaux sur le Lazarillo, la Célestine ou Don Quichotte, l'histoire du juif errant, la question de l'honneur ou la fonction du pícaro... Ce qui relève de la manière propre à M. Blanco, c'est qu'elle n'est jamais strictement descriptive : $M$. Blanco réinvente, à la lumière de ses préoccupations scientifiques, de son grand savoir et de son énergique intelligence, les textes et études qu'elle analyse.

4 Je n'évoquerai ici que deux moments de sa présentation. Le premier concerne la double rencontre, précoce et concomitante, de Bataillon avec Unamuno et Érasme. En 1923, paraît sa traduction de En torno al casticismo (L'essence de l'Espagne) et en 1924, le Bulletin Hispanique publie l'un de ses premiers articles : "Érasme et la Chancellerie Impériale ». Cette coïncidence n'est pas seulement signalée, elle inspire à $\mathrm{M}$. Blanco une réflexion lumineuse (p. 35-38) : entre Érasme l'humaniste qui «domine l'Empereur» par sa "souveraineté spirituelle», Unamuno qui «voudrait concilier l'amour d'une Espagne éternelle, dont le mysticisme serait la cristallisation la plus pure, et l'appel à une métamorphose, à une régénération [...]» et le jeune Marcel Bataillon qui cherche «dans le passé un modèle de l'intellectuel » et s'intéresse à l'essence de l'Espagne, s'instaurent de nouvelles affinités électives : «[...] l'article sur "Érasme et la Chancellerie impériale" est le pendant, en ces premières années vingt, de la traduction de En torno al casticismo » (p. 37). Un monde s'ouvre...

5 Le deuxième moment concerne l'article de $1941^{3}$ dont la première partie détaille « les formes que prend en Espagne la légende du juif errant » et dont la deuxième décrit le procès inquisitorial d'un usurpateur, un faux Juan Desperandios, en 1547. L'interprétation, très créative, de $\mathrm{M}$. Blanco s'empare de l'article, le bouleverse, l'illumine et lui permet de définir la « méthode Bataillon » : pour « élucider une énigme 
littéraire », il s'appuie sur une enquête philologique et «les magnifiques documents bien espagnols que gardent les archives inquisitoriales" en pratiquant "d'habiles tissages entre la documentation historique et les textes », eux-mêmes appréhendés " en se rapprochant le plus possible de la vision que les acteurs de l'histoire et les auteurs des textes avaient d'eux-mêmes [...] », ce que Bataillon appelait « le sens littéral ».

6 L'activité pédagogique de Bataillon, avant son entrée au Collège de France, est évoquée à la faveur de la découverte par Hélène Marché, directrice de la bibliothèque MarcelBataillon, de liasses de cours autographes, rédigés entre 1939 et 1944, en pleine guerre. À partir d'eux, Mercedes Blanco peut mesurer l'investissement de Bataillon dans l'enseignement qu'il a dispensé par temps de guerre, le « délassement enchanteur » que ces cours procurent à la lecture, et la distance qui les sépare de l'activité de recherche : pas de données nouvelles, "pas de créations géniales "- à cause sans doute de l'impossibilité de voyager et de travailler dans les archives - mais une réflexion remarquable sur des faits et des ouvrages connus. Et tout au long de la variation, c'est le respect fasciné de $\mathrm{M}$. Blanco pour un hispaniste exceptionnel qui restitue à Marcel Bataillon son inextinguible magistère.

7 Face à l'attitude pacifiste de Bataillon pendant la guerre (p. 33) ${ }^{4}$, c'est sur le " refus absolu » (p. 74) opposé par Jean Cassou au fascisme et sur son engagement de résistant que Maria Zerari ouvre sa Variation: "Jean Cassou, hispaniste. Sur Le Gréco (1931) et Cervantes (1936)» (p. 73-120). À la lecture des quatre parties de son essai : I- Critique et domaine espagnol ; II - Traduire Cervantès : circonstances et manière ; III - Le Gréco : manière, situation, vision et IV-Cervantes: un Cervantès synthétique, j'ai été frappée par deux choses : les rythmes de l'écriture de Maria Zerari et la puissance qu'y acquiert un détail, en apparence infime.

8 Commençons par le détail : la virgule qui, dans le titre, sépare 'hispaniste' de 'Jean Cassou'. Ce que la virgule met en relief est une désignation, l'un des mots de Cassou « à "épingler comme un papillon de sens " (p. 75), et un titre définitif. Cassou, même s'il est aujourd'hui presque oublié ${ }^{5}$ et s'il se désignait lui-même comme 'hispanisant', a été un hispaniste comme il en existe peu: grand lecteur de poésie et de littérature espagnoles, ébloui par une "langue royale, faite pour la poésie», habité par une "bibliothèque choisie" mais nombreuse (p.79), en permanence au fait de ce qui s'écrivait et se pensait en Espagne, « introducteur, traducteur, ambassadeur » (p. 79) en France des écrivains espagnols les plus importants, mais aussi «figuratif» (p. 98), " amant des formes " (p. 99) au vaste et singulier musée intérieur, ce sont ses travaux sur Cervantès et Le Gréco (ces deux " solitaires », ces deux « "rénovateurs" animés par une même liberté créatrice», p. 20-21) ainsi que sa traduction du Quichotte qui le consacrent hispaniste à part entière. Car il est aussi romancier et poète, chroniqueur, essayiste, inspecteur des arts appliqués, rédacteur en chef d'Europe, conseiller de Jean Zay, Compagnon de la Libération, conservateur en chef du futur Musée national d'Art Moderne, etc.

9 C'est là qu'interviennent les rythmes de l'écriture de M. Zerari, qui entraînent successivement le lecteur dans les «circonstances » et les «manières » dont Cassou, « sous le signe d'une farouche volonté de réhabilitation culturelle» (p. 20) - qui a été, pourrait-on ajouter, celle de tous les premiers hispanistes - valorise « pour un lecteur français de sa génération [...] un "humanisme espagnol" » (p. 95). Après des pages très vives où s'accumulent références, informations et faits contextuels, la méditation 
s'arrête et s'approfondit sur l'objet commenté, l'ensemble visant ainsi à une sorte de riche et restreinte « exhaustivité ».

L'évocation de Cassou traducteur de Cervantès est l'exemple le plus net de la «manière Maria Zerari »: si, dès les années 20, Cassou consacrait son activité de traducteur et d'essayiste à Cervantès, c'est qu'il avait saisi, en profondeur, après le tricentenaire de la Première partie (1905), la formidable focalisation sur le Quichotte des questionnements, des remises en cause et aspirations des écrivains et des philosophes espagnols. En parallèle, du côté français, un «quichottisme» diffus, qui renouvelait les préoccupations des universitaires, avait suscité plusieurs retraductions du Quichotte (p. 82-85) de sorte que, pour cet impressionnant capteur et acteur de la vie intellectuelle hispano-française qu'était Cassou, l'air du temps s'en trouvait comme " quichottisé ». Faisant suite à une somme de faits, de noms, d'idées, c'est ensuite au cœur du travail solitaire d'un homme, intérieurement habité d'une foule de lectures et de traductions, que le lecteur est amené à séjourner pour y réfléchir à ses options archaïsantes et au travail de re-création d'un "réviseur » de grand talent. Jusqu'au moment où quelque trois pages d'échantillons de sa traduction (90-92), séduisante par sa fluidité limpide, captivent le lecteur, lui font entendre le texte espagnol sous les mots français et l'expédient irrésistiblement vers le texte original et vers d'autres traductions, tant le presse l'envie de «comparer » et de lire et relire, dans une quête sans fin, à la Borges, l'entier du palimpseste. La présentation de Maria Zerari, on l'aura compris, honore non seulement cet «hispanista de raíz» qu'a été Jean Cassou, mais l'hispanisme universitaire français qui le reconnaît comme l'un des siens.

11 Autre grande figure de l'hispanisme français, mais aussi nouveau défi pour son présentateur, Roland Béhar. Le Siècle d'or d'A. Rumeau, ultérieur à sa thèse sur Larra, c'est El Abencerraje, Góngora et surtout le Lazarillo, dont il avait conçu le grand projet d'une édition critique dès 1954, à Bordeaux, où il occupait la chaire d'Espagnol depuis 1953 et où il dirigeait le Bulletin Hispanique. Mais c'est à compter de son arrivée à la Sorbonne (1955) qu'il publie, dans cette revue et dans celle des Langues néo-latines ses "notes ", réflexions et travaux sur le Lazarillo. Malheureusement, le projet d'édition critique n'a pas abouti et ce n'est qu'après sa mort, en 1993, qu'à l'initiative d'Augustin Redondo ont été regroupés et publiés, sous le titre de Travaux sur le "Lazarillo de Tormes", les quatorze études, d'une richesse et d'une précision remarquables, qu'A. Rumeau a consacrées aux problèmes textuels du Lazarillo, à la transmission du texte et à une proposition d'interprétation et d'attribution du petit chef d'œuvre.

A. Rumeau est resté "un peu à l'ombre ", écrit R. Béhar (p. 138) : il l'explique par les difficultés liées au projet d'édition lui-même autant que par un attachement trop respectueux aux Maîtres, Bataillon et, avant lui, Morel-Fatio. Mais ce qui ressort de la puissante dramatisation, orchestrée par R. Béhar, de cette mise en retrait, dans la partie II de la variation: "La fascination du Lazarillo", c'est que Rumeau n'avait pas prévu la pujanza de la jeune érudition espagnole et de certains hispanistes français. Les fragments consacrés à son travail y sont littéralement 'enclavés' entre les contraintes de ses cours d'agrégation, l'impressionnant magistère de Bataillon et la publication des éditions du Lazarillo de José Caso González et de Francisco Rico en 1967, suivies de la traduction de Molho dans la Pléiade en 1968. Moins enclavée qu'affrontée, la question des premières éditions du Lazarillo (p. 130) doit faire face aux remises en question de la recherche ultérieure (Caso, Rico et Ruffinatto) alors que la question de l'auteur du Lazarillo, non refermée, à laquelle Rumeau apporte sa propre hypothétique réponse 
(Hernán Núñez, le Comendador griego) lui vaut trois amples pages qui ont la saveur douce-amère du semi-échec.

On peut dès lors saluer le "Rumeau » de Roland Béhar comme la tentative réussie de « rendre justice bibliographique» (note 11, p. 124) au rayonnement d'un professeur et d'un chercheur exemplaires, unanimement reconnu par ses collègues et les générations d'hispanistes qu'il avait formés et qui sont tous cités dans «IV- Un legs méconnu ». Le titre de la variation de R. Béhar : «Littérature et contresens. Aristide Rumeau ou le souci de l'exactitude ", s'éclaire à la lecture de l'idée, exprimée par Rumeau lui-même, selon laquelle « les lecteurs sont tous des traitres et la littérature vit du contresens » (p. 145), un contresens que, dans son enseignement comme dans ses travaux, il s'était en permanence efforcé d'éviter au prix d'un souci «d'exactitude » qui a peut-être bridé l'audace nécessaire aux productions de la recherche.

14 C'est en quatre volets («I- Faire connaître le théâtre classique », « II - Redécouvrir le théâtre de cour ", «III - Calderón en Sorbonne à l'aube de mai 68 : quelle nouvelle critique ? » et «IV - L'ouvrage sur la Comedia ») que Florence d'Artois et Yves Germain dressent le portrait d'un hispaniste d'action. L'énergie que Charles V. Aubrun a déployée avec Robert Ricard pour donner à l'Institut Hispanique ${ }^{6}$ sa taille et son importance actuelles, et avec Robert Marrast pour faire connaître à un public français, parfois très réticent (p. 149), les pièces du répertoire classique espagnol, on la retrouve dans ses propositions théoriques et ses prises de position « contre » le nouvel horizon de l'analyse des textes autour de 1968.

Fondateur du Théâtre espagnol à la Sorbonne, il édite des comedias avec José F. Montesinos et, pionnier, il (re)découvre le théâtre de cour, genre autonome qu'il contextualise et dont il capte le caractère "radicalement nouveau» (p.151). Plus encore, la modernité d'Aubrun s'impose dans sa claire perception de la polyphonie et de l'ambigüité du corpus, même si, pour La estatua de Prometeo, l'élucidation "des différentes couches de sens de la pièce: sens historique, sens moral, sens psychologique, sens allégorique », paraît «scolaire» ou "paradoxale» (p. 152) aux auteurs. Or, cette élucidation doit beaucoup à la Philosofía secreta de Juan Pérez de Moya, d'ailleurs revendiquée par Aubrun lui-même : «Nous adoptons la méthode de critique des textes en vigueur au XVII ${ }^{\mathrm{e}}$ siècle. On en cherchera les règles dans la Philosophia secreta de Pérez de Moya ${ }^{7}$, qui les reprend de Léon Hébreu » (p. 155-56).

J'en viens à la partie la plus enlevée, la plus tonique de la variation, la II, que j'appellerais celle du vorrei e non vorrei d'Aubrun. Elle réussit à incarner, en sa figure, la résistance et les tergiversations des tenants d'un conservatisme ou d'un réformisme prudents en matière de lecture des textes (p. 156-57), à un moment où l'exceptionnelle irruption de propositions nouvelles les bouscule et les irrite. On y retrouve deux des qualités d'Aubrun: celle de capteur curieux et éclectique de "l'air du temps", qu'il rejette de toutes ses forces mais qu'il respire, et celle de l'homme d'action. Qu'on relise l'étonnant extrait de sa préface à la seconde édition de Eco y Narciso, suivi d'un discret commentaire des auteurs (p. 154-55) : on y découvre - à propos de la méthode d'analyse textuelle - le lexique d'un chef militaire et celui d'un explorateur ou d'un arpenteur, curieusement mêlés à celui de la sémiotique.

Et ses recommandations pour rendre compte d'une œuvre (les nécessaires contextualisation, structure, "affrontements au sein de notre psyché») semblent frôler, mais superficiellement et sans les nommer, les approches nouvelles : marxiste, structuraliste et psychocritique. On n'aurait garde d'oublier l'approche sémiotique ou 
littérale, celle du «texte nu, ce qu'il dit stricto sensu» (p. 155), mais sans que soient véritablement franchis le ou les pas théoriques. Le débat n'était pas que parisien et je sais gré aux auteurs de la variation d'en avoir incarné les arguments, avec autant de vivacité, dans leur portrait de C. V. Aubrun.

«Les variations Molho » de Jean-Pierre Étienvre, une fois franchie « l'ouverture » dans laquelle la malice du présentateur fait un vivant écho à la malice, réjouissante et communicative, du re-présenté, mettent immédiatement en lumière un paradoxe : la puissante unité d'une pensée théorique et analytique tendue vers la recherche d'une vérité est inséparable d'un renouvellement constant des questionnements auxquels Maurice Molho soumet les textes. Limitées pour ce qui est des 'objets' à un va-et-vient fructueux entre linguistique et littérature, les variations Molho se multiplient et se diversifient, dans le champ de la littérature, au gré des avancées théoriques qu'il assimile avec autant de voracité que de passion et de sûreté de jugement.

Sans négliger le tracé de la carrière de M. Molho (p. 160) ni l'apport exceptionnel que constitue la "monumentale édition critique du Fuero de Jaca [...]» (p. 161), sa thèse, Jean-Pierre Étienvre orchestre brillamment sa fracassante entrée en littérature et en littérature du Siècle d'Or avec l'article Soledades, publié en 1960 dans le Bulletin Hispanique. Il est concevable qu'avec la thèse, ce texte ait été décisif pour que Noël Salomon recrute Molho à Bordeaux, en 1960, en qualité de chargé de conférences sur la Chaire de Philologie hispanique. "Dans cet article pionnier - écrit Étienvre-, une avancée fondamentale est accomplie en direction de la "poétique" au détriment de l'histoire littéraire » (p.161-62) : c'est cette avancée, alors extraordinaire, qu'il m'a été donné de vivre comme étudiante jusqu'en 1964, puis comme assistante et maîtreassistant jusqu'en 1969.

À partir de là, l'activité de Molho siglodorista se déploie dans les deux champs de la traduction et de la littérature. Tous ses travaux - il le revendique - sont ceux d'un linguiste qui observe les faits et les textes au pied de la lettre, et sa méthode, il la veut « rigoureusement littérale ». Étienvre rappelle les grandes traductions de 1968 pour la Pléiade (Romans picaresques espagnols), celle de 1970, pour Aubier-Flammarion, du Casamiento engañoso y Coloquio de los perros et celle de 1994, pour José Corti, du chant du cygne de Cervantès: Los trabajos de Persiles y Sigismunda. Mais ce qu'il met remarquablement en lumière c'est la théorisation à laquelle Molho soumet le (ou son) travail de traducteur, produisant d'importants « discours de la méthode » (je cite) sur sa pratique.

21 S'en tenant principalement aux faits, Étienvre rappelle que Molho ne cesse de réfléchir à (et de publier sur) la métaphore baroque, le concepto ou une théorie du cultismo. Les rencontres avec de grands esprits, que lui valent ses incessantes lectures, croisent alors les poètes, dramaturges et auteurs de narrations, objets de ses théorisations successives : pour n'en citer que quelques-uns, je rappellerai Descartes, Freud, Karl Abraham, Marx, Gramsci, Soriano, Caro Baroja, Bataillon, Salomon, Amado Alonso..., qui fécondent ses prodigieuses intuitions sur les Quevedo, Tirso de Molina, Calderón, Gabriel Bocángel et, par-dessus tout, Cervantès dont (cité par Étienvre) il écrivait : « un solitario que si tomaba la pluma y escribía un libro era leído por cantidad de lectores que formaban no un público sino una inmensa voz escuchante »: la beauté de cette trouvaille (Moho n'aimait pas les notions de «chercher» ou de «chercheur»: lui, il "trouvait») rayonne dans les pages qu'Étienvre dédie à Molho cervantiste et à 
l'introduction «Leer a Cervantes » du recueil de ses travaux, De Cervantes, publié bien après sa mort, en 2005.

S'il s'en tient aux faits, Étienvre, qui connaissait bien le personnage et son «alchimie interprétative » (p.166), frappe bref mais juste lorsqu'il cite : «Yo hago siempre una lectura de lingüista, que no perdona nada, porque es literal » (ibid.). No perdonaba nada, don Mauricio, et c'était tout ensemble exaltant au plus haut point, exaspérant et admirable. Molho - rappelle Étienvre - revendiquait explicitement «le droit à l'erreur » (note 22, p. 165). Je lui ai connu une autre formule par laquelle il mettait en lumière le rôle capital de l'erreur (Felix culpa, chante l'hymne Exultet jam angelica turba ...) dans la découverte de la ou d'une vérité : «il y a des erreurs à bonne pente », les autres, celles à mauvaise pente, étant radicalement exclues de son univers. À ce propos, la phrase citée p. 167 : «el lenguaje tiene un espesor que hace que signifique a niveles cada vez más profundos de sí mismo ", n’a eu qu'un temps. Dans ses travaux ultérieurs de linguistique, au sein de la Molache, Molho renoncera à l'idée longtemps admise que le verbe, passant de l'indicatif au subjonctif, "descende au-dessous de lui-même ». Mais l'erreur est à bonne pente et elle permet de comprendre que ce sont les strates de notre propre " épaisseur ", variable, que le langage anime et ce sont elles qui font qu'il puisse signifier à des niveaux chaque fois plus profonds de nous-mêmes.

Tel a été le magistère de M. Molho, dont j'ai eu le bonheur d'être « l'élève ». Je remercie très vivement Jean-Pierre Étienvre de m'avoir associée à sa présentation et d'avoir restitué, au-delà de l'anecdote (et la vie universitaire de Molho n'en manquait pas), les belles et grandes lignes de force d'une pensée toujours au-dessus, toujours originale et toujours féconde.

\section{NOTES}

1. Dans Les cultures ibériques en devenir. Essais publiés en hommage à la mémoire de Marcel Bataillon (1895-1977), Fondation Singer-Polignac, 1979: «L'itinéraire intellectuel de Marcel Bataillon. Du sens littéral à la métahistoire » (p. 57-120), I, Aux carrefours de l'humanisme européen (p. 61), Un philosophe hédoniste (p. 62), «Thèmes et variations sur un Siècle d'Or » (p. 69-85). Je souligne.

2. La consultation de la collection ancienne (1899-2007), sur Persée, permet de mesurer cet impact. On peut signaler à titre (aujourd'hui) anecdotique les 212 références de $\mathrm{M}$. Bataillon, les 39 d'A. Rumeau, les 318 de C.V. Aubrun et les 38 de M. Molho, auxquelles il faudrait ajouter les publications ultérieures à 2007.

3. «Pérégrinations espagnoles du juif errant », Bulletin Hispanique, vol. XVIII, 1941, p. 81-122.

4. Plus radicalement pacifiste qu'Érasme lui-même, Bataillon avait commis l'erreur, largement partagée, de se joindre à ceux «qui voulaient la paix au prix de complaisances aveugles envers Hitler », erreur qu'il avait, par la suite, beaucoup regrettée (Blanco, p. 31).

5. Dans la note 1, M. Zerari signale la publication, dans le numéro 1063-1064 de la revue Europe (organe de l'anti-fascisme dont il avait été le rédacteur en chef en 1936) d'un dossier Jean Cassou. Elle n'a pu rendre compte de ce dossier, paru en novembre-décembre 2017, alors qu'elle avait fini de rédiger son article. Outre les hommages rendus au résistant et au poète, il faut y lire l'étude pénétrante et lucide de Jean-Marc Pelorson sur le «tropisme hispanique » de Jean Cassou dont la 
langue d'écriture était exclusivement le français: "L'entre-deux franco-hispanique de Jean Cassou » (p. 196-204).

6. À la même époque, à Bordeaux et après le départ d'A. Rumeau et de Charles V. Aubrun pour la Sorbonne, Noël Salomon, "se refusant à céder à la "parisianite" ", faisait de l'Institut d'Études Ibériques et Ibéro-Américaines un centre susceptible d'attirer durablement des Maîtres (M. Molho a été l'un d'eux) et des générations d'étudiants (M. Chevalier, "Noël Salomon (1917-1977) », Bulletin Hispanique, 1977, 79-1-2, p. 11-15).

7. Capítulo II, « De los sentidos que se pueden dar a una fábula ».

\section{AUTEURS}

\section{NADINE LY AGUILA}

Université Bordeaux Montaigne 